\title{
Research on Spatial Dependence of Regional Highway Network and Economic Development
}

\author{
Beibei $\mathrm{GAO}^{\mathrm{a}, \mathrm{b}, \mathrm{c}}$ and Shijie $\mathrm{WANG}^{\mathrm{a}, \mathrm{b}, \mathrm{c}, 1}$ \\ ${ }^{a}$ Faculty of Geomatics, Lanzhou Jiaotong University, Lanzhou, Gansu, 730070, China \\ ${ }^{\mathrm{b}}$ National-Local Joint Engineering Research Center of Technologies and Applications \\ for National Geographic State Monitoring, Lanzhou, Gansu, 730070, China \\ ${ }^{\mathrm{c}}$ Gansu Provincial Engineering Laboratory for National Geographic State Monitoring, \\ Lanzhou, Gansu, 730070, China
}

\begin{abstract}
In order to simplify the decision-making of highway construction planning, GIS spatial analysis method and mathematical statistics method are combined to establish a mathematical model of the spatial dependence relationship between highway density and economic development indicators. The model can explain $79.8 \%$ of the highway development demand in Gansu Province, and has good accuracy and reliability. The model is used to check the density of highway network in the planning year, which can evaluate the adaptability of highway network construction and economic development, so as to simplify the pre-feasibility study of highway engineering.
\end{abstract}

Keywords. Spatial analysis, highway network spatial dependence model, highway pre-feasibility study.

\section{Introduction}

The traditional economic data integration method is still adopted for feasibility evaluation in the pre-feasibility study stage of highway construction in China. This paper analyzes the relationship between regional economic development indicators and highway network density, aiming to put forward a technical model that can assist highway pre-feasibility study decision.

The speed and quality requirements of China's highway development are far higher than the status quo. It is demanded by national development at this stage to simplify all links of highway network construction and improve work efficiency. Wei Wei, Shi Peiji, Demin Yong, et al, [1, 2]. The expansion law model of highway network density and its spatial dependence were studied. The fitting results of highway network expansion law have certain reference value for current highway network planning, but data and method still need to be updated to adapt to the current road network development situation.

The spatial distribution characteristics of regional highway network construction, population and GDP were quantitatively analyzed by using GIS spatial analysis

\footnotetext{
${ }^{1}$ Shijie Wang, Faculty of Geomatics, Lanzhou Jiaotong University, Lanzhou, Gansu, 730070, China. e-mail: wangshijie@mail.lzjtu.cn
} 
method. Combined with the current situation of highway development in Gansu Province, the mathematical model is established.

\section{Data and Spatial Analysis}

\subsection{Research Data and Preprocessing}

The relevant statistical data of this paper (2014-2019a) are from the website of Gansu Provincial Bureau of statistics. Road network data (2014-2019a) is from open street map (OSM). According to highway classification and highway route design specification (JTG D20-2017), the highway mileage equivalent conversion coefficient bi is calculated. The calculation results of highway mileage equivalent conversion coefficient are shown in Table 1.

Table 1. Equivalent conversion coefficient of different Highway grade

\begin{tabular}{lllllll}
\hline Grade & Expressway & First class & Secondary & Third & Class IV & Extraneous \\
\hline bi & 4.104 & 3.488 & 1 & 0.199 & 0.099 & 0.004 \\
\hline
\end{tabular}

The statistical GDP, population data, equivalent road network length conversion data and administrative division vector data are superposed and analyzed. The density calculation and inverse distance interpolation analysis are used to complete the data preprocessing.

\subsection{Data Space Analysis}

The spatial distribution map of highway network density is obtained by inverse distance weighting method (as shown in Figure 1).

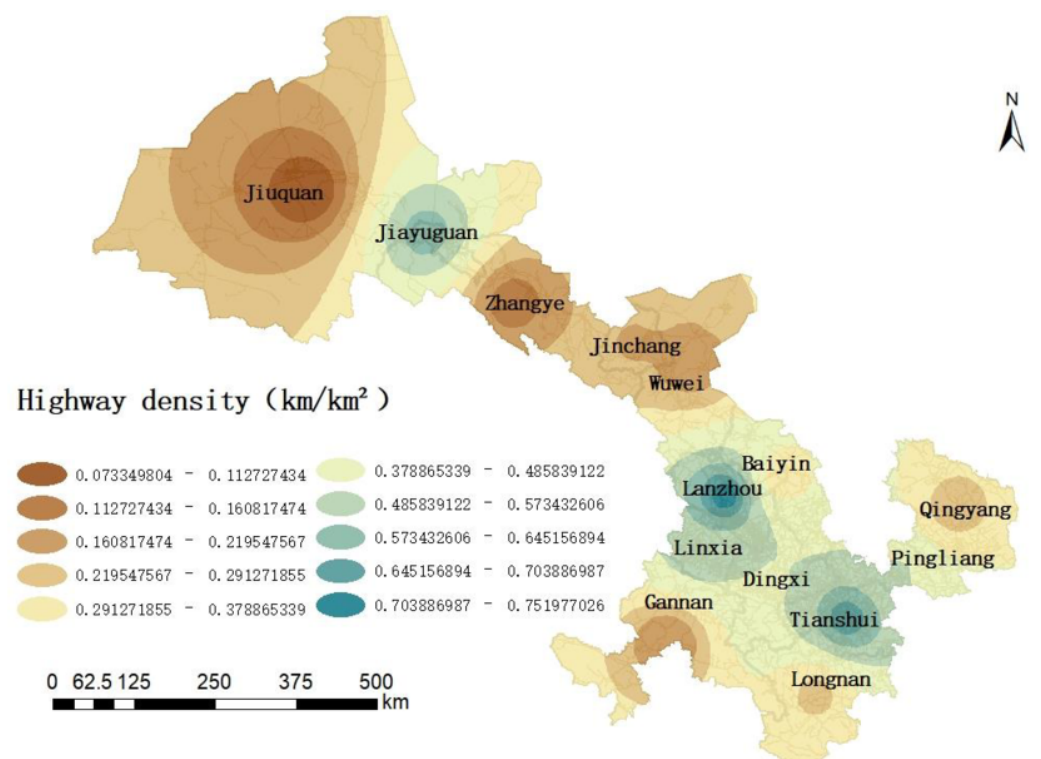

Figure 1. Spatial distribution of highway network density in 2019 
Using ArcGIS to calculate the population density and carry out inverse distance interpolation analysis, the spatial distribution map of population density in 2019a is obtained (as shown in Figure 2).

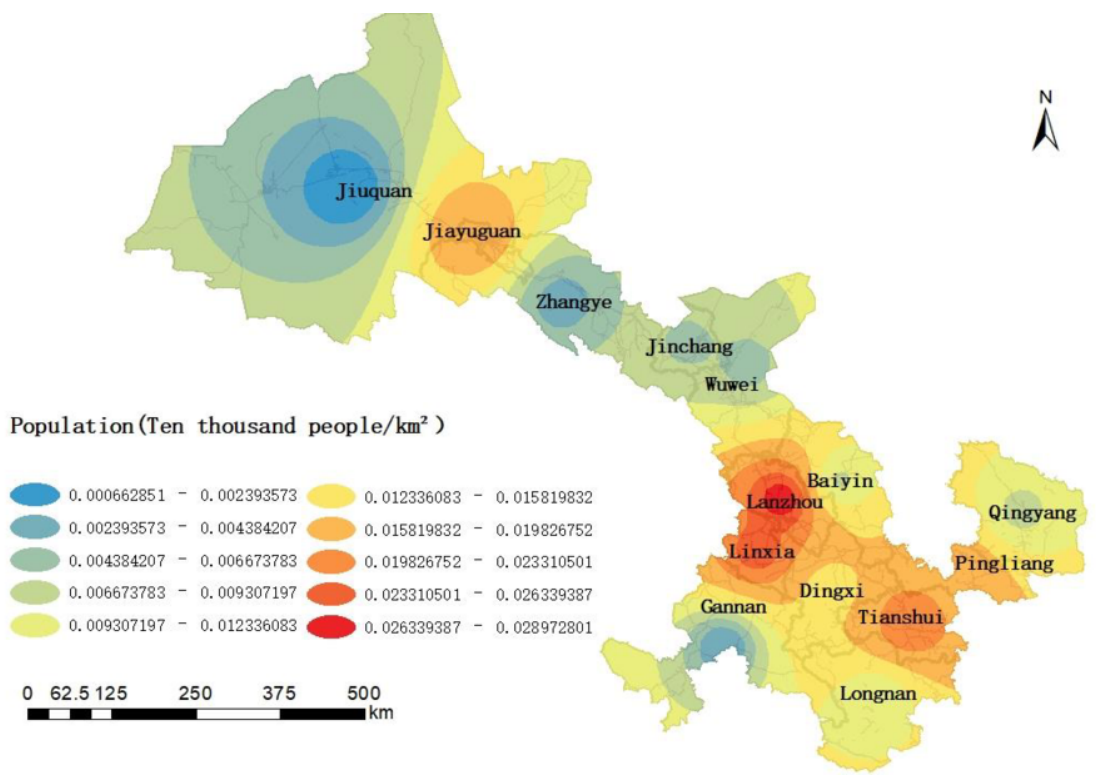

Figure 2. Spatial distribution of population density in 2019

The regional GDP indicators include GDP, GDP density (GDP per square kilometer) and per capita GDP, [3, 4]. The correlation between GDP indicators and road network density is drawn, (as shown in Figure 3).

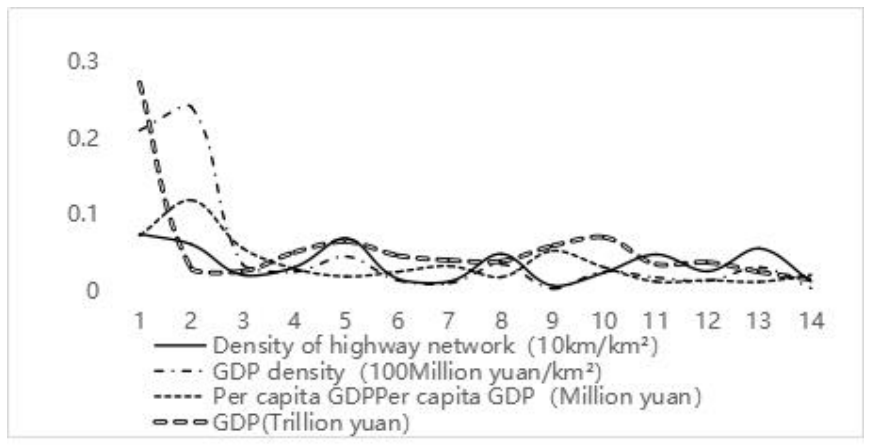

Figure 3. Comparison of GDP indicators with road network density

As shown in the Figure 4, Abscissa 1-14 in the figure indicates Lanzhou, Jiayuguan, Jinchang, Baiyin, Tianshui, Wuwei, Zhangye, Pingliang, Jiuquan, Qingyang, Dingxi, Longnan, Linxia Hui Autonomous Prefecture and Gannan Tibetan Autonomous Prefecture from the frequency, amplitude, extreme point synchronization, maximum value analysis, discount fitting effect analysis, the regional GDP density has the best correlation with road network density, and GDP density is selected as another influencing factor of road network construction planning model. The 
distribution map of GDP density in Gansu Province in 2019a is drawn (as shown in Figure 4).

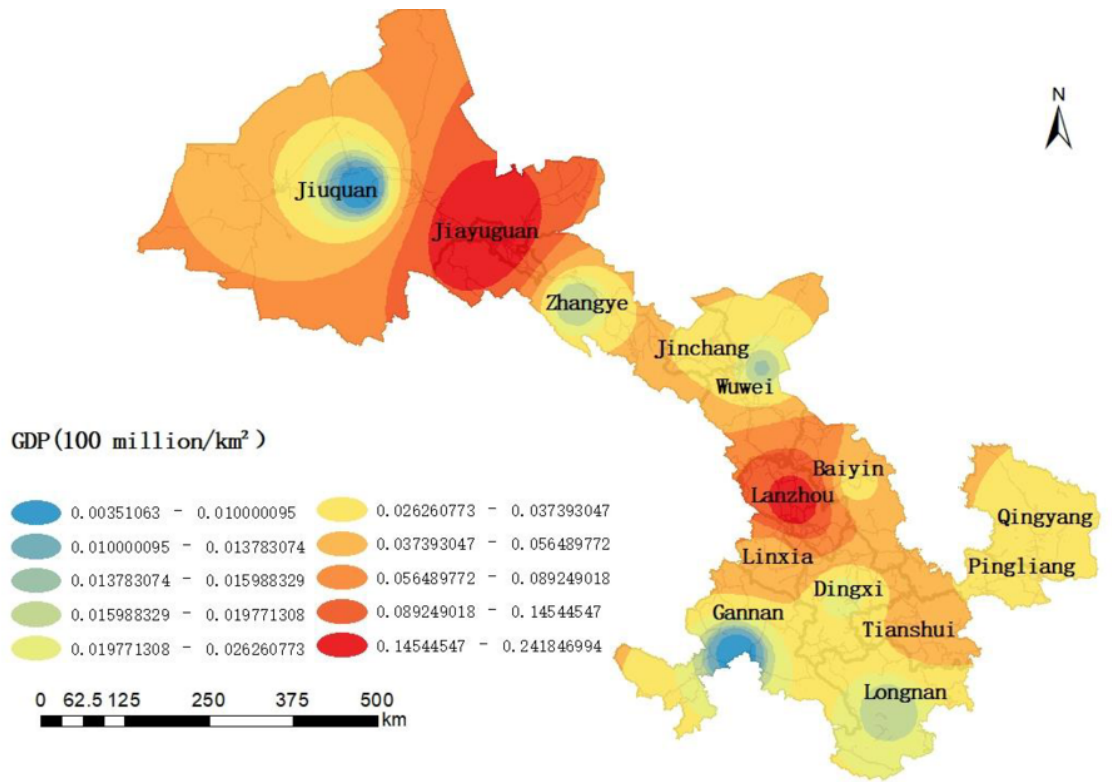

Figure 4. Spatial distribution of GDP density in 2019

The density of highway network, population density and GDP density have logical correlation and spatial characteristics of density distribution. The specific performance is as follows: the region with higher highway network density in Gansu Province is also the center of gravity of population density and GDP density; the three have the spatial distribution characteristics of regional government location as the center of gravity. Therefore, highway network density, GDP density, population density as the basis of road network construction planning model are reliable.

\section{Correlation Quantification}

The spatial correlation degree of geographical elements is described quantitatively, and the calculated value of correlation coefficient is used as the basis of quantitative analysis. The correlation coefficient is defined as:

$$
r_{x y}=\frac{\sum_{i=1}^{n}\left(x_{i}-\bar{x}\right)\left(y_{i}-\bar{y}\right)}{\sqrt{\sum_{i=1}^{n}\left(x_{i}-\bar{x}\right)^{2} \sum_{i=1}^{n}\left(y_{i}-\bar{y}\right)^{2}}}
$$

Among them, $n$ is the number of survey samples, $\bar{x}, \bar{y}$ are the average of variables $x$ and $y . r_{x y}$ is the correlation coefficient, and its numerical value describes the correlation between variables $x$ and $y, r_{x y}>0$ indicates positive correlation; $r_{x y}<0$ 
indicates negative correlation; the absolute value of $r_{x y}$ is close to 1 , the closer the correlation between the two variables $x$ and $y$; the closer to 0 , the less close the relationship between the two elements. SPSS software conducts correlation analysis based on this principle. Based on the highway network density, population density and GDP density of 14 cities and prefectures in Gansu Province from 2014 to 2019a, an analysis sample with 84 samples and the degree of freedom is 2 be established, and the correlation coefficient is analyzed and calculated by SPSS, Table 2.

Table 2. Correlation coefficient table of highway density and economic development index

\begin{tabular}{rrrr}
\hline Element index & \multicolumn{1}{l}{ Highway } & \multicolumn{1}{l}{ GDP } & \multicolumn{1}{l}{ Population } \\
\hline Highway & 1.000 & 0.849 & 0.819 \\
GDP & 0.849 & 1.000 & 0.861 \\
Population & 0.819 & 0.861 & 1.000 \\
\hline
\end{tabular}

\section{Highway Network Depends on Regression Fitting of Economic Development Law}

According to the results of correlation coefficient analysis, without considering the influence of policy preference and resource industrial structure on highway network density, GDP density and population density are the main influencing factors of highway network expansion. Based on the highway network density, population density and GDP density of 14 cities and prefectures in Gansu Province from 2014 to 2019a, an analysis sample with 84 samples and the degree of freedom is 2 be established to conduct regression fitting on the spatial dependence relationship between highway density and economic development in Gansu Province.

\subsection{Theoretical Model}

According to the theory of Gerber Douglas production function [5], it is assumed that the theoretical model of spatial dependence of highway density and economic development in Gansu Province is as follows:

$$
D_{r}=K P^{a} G^{b}
$$

Among them, $D_{r}$ is the density of regional highway network; $P$ is the population density; $G$ is the GDP density; $K, a, b$ are the coefficient indexes of social development, population density and economic progress. $D_{r}, P$ and $G$ can be obtained from regional statistical data. $K, a$ and $b$ need to estimate the parameters through regression analysis, and take the natural logarithms on both sides of formula (2):

$$
\ln D_{r}=\ln K+a \ln P+\ln G
$$


order $Y=\ln D_{r}, \quad A=\ln K, \quad X_{I}=\ln P, \quad X_{2}=\ln G$, then the above formula can be written as follows:

$$
Y=A+a X_{1}+b X_{2}
$$

\subsection{Model Regression Fitting}

Based on the statistical data of highway network density, population density and GDP density of cities and prefectures in Gansu Province from 2014 to 2019a, combined with the bidirectional interaction between the three statistical data, the fitting model of spatial dependence between highway density and economic development in Gansu Province is obtained:

$$
\ln \left(D_{r}\right)=1.403+0.351 \ln P+0.284 \ln G
$$

The fitting degree of SPSS analysis equation $\mathrm{R}^{2}=0.803$, after adjustment $\mathrm{R}^{2}=$ 0.798 , the explanatory variable can explain $79.8 \%$ of the dependent variable changes; the correlation coefficient between the fitting model and the dependent variable is 0.961 , the absolute value of the correlation coefficient is very close to " 1 ", and the regression equation is highly correlated with the analysis variable; sig value of 0.00 is less than 0.05 , indicating that the regression effect is significant and meets the simulation requirements. The function of highway network density, GDP density and population density in Gansu Province is obtained by exponential operation on both sides of equation (5):

$$
D_{r}=4.0674 P^{0.351} G^{0.284}
$$

Using the fitting model of spatial dependence between highway network density and economic development in 2014-2019a, the density demand of highway network in 2019a is calculated with the city level division as the unit, and the regional GDP density data and population density data at the end of $2018 \mathrm{a}$ are used as known variables to calculate the density demand value of highway network in Gansu Province in 2019a. Draw the comparison chart of the actual built road network density and the demand value of road network density at the beginning and the end of the year in Gansu Province in 2019a (as shown in Figure 5), Note: Abscissa 1-14 in the figure indicates Lanzhou City, Jiayuguan City, Jinchang City, Baiyin City, Tianshui City, Wuwei City, Zhangye City, Pingliang City, Jiuquan City, Qingyang City, Dingxi City, Longnan City, Linxia Hui Autonomous Prefecture and Gannan Tibetan Autonomous Prefecture. 


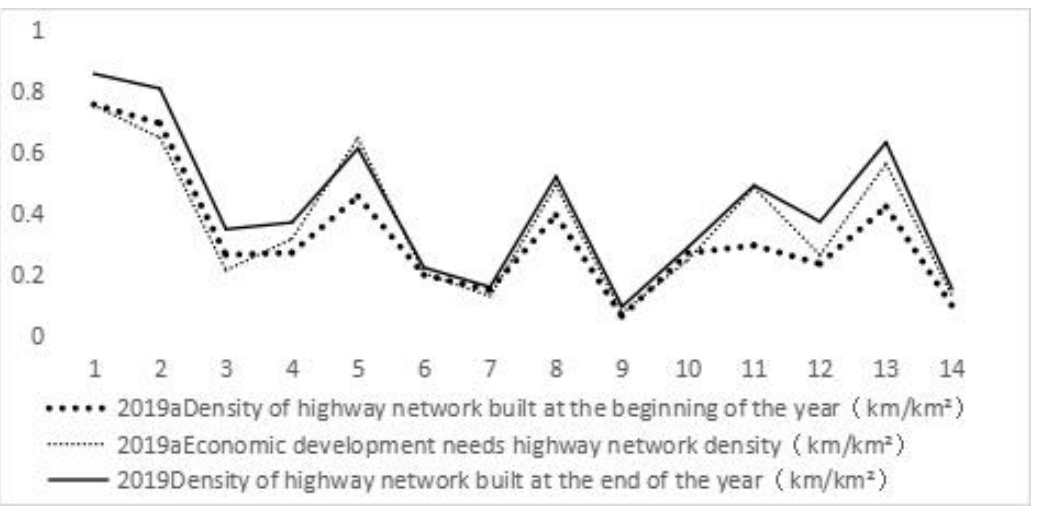

Figure 5. Comparison between the actual density of completed road network and the demand value of road network density at the beginning and end of the year in Gansu Province in 2019

According to the calculation results of the model, the pre-feasibility study of highway network construction in Gansu Province was made at the beginning of 2019a. Except Lanzhou City, Jiayuguan City and Jinchang City, the demand value of road network in other 11 cities and prefectures was higher than the built value. The highway network built in Gansu Province at the beginning of the year was not enough to meet the demand of economic development for highway network construction at that time, and 11 cities and states needed different degrees of highway network densification compared with the beginning of the year, the density of the highway network has generally increased, which can meet the needs of economic development at the beginning of the year, but the regional development is not balanced, and the road network construction work of some cities and prefectures, especially Tianshui City, it still needs to be strengthened. As for the economic development situation at the end of 2019a, the statistical data will still play an auxiliary role in decision-making for the construction planning of 2020a highway network Use.

\section{Conclusion}

The regression fitting results show that there is a significant correlation between the density of highway network, population and GDP. The density of GDP, highway network construction and population interact in the interaction circle. Based on the fitting calculation of economic data and highway network data from 2014 to 2019a, the calculation model of spatial dependence relationship between highway network density and economic development in Gansu Province is given. Through verification, the model has high fitting degree, which can assist the feasibility evaluation of regional new highway projects in Gansu Province. The following directions can be further studied: (1) whether there is a similar correlation law in changing the research area; (2) changing GDP and population measurement indicators (such as per capita GDP), what is the correlation between weighted population density and regional highway network density; (3) whether other correlation factors (climate, topography, etc.) can be added to expand the interaction circle of highway network construction density to better assist the highway pre-feasibility study; (4) whether there are other regression algorithms to make the model fitting more accurate. 


\section{Acknowledgments}

This article is one of the phased achievements of the National Natural Science Foundation of China Project "Research on the Accuracy of Existing Wired Track Detection Based on UAV” (41861061), supported by LZJTU EP 201806.

\section{References}

[1] Wei W, Shi PJ, Tuo MY, et al. GIS-based analysis of density distribution characteristics and spatial dependence of road network in Gansu Province, Geoscience, 2012, 32(11): 1297-1303.

[2] Wei W, Shi PJ, Shi BB, Lei L, Zhou JJ. Analysis of the spatial organization characteristics and dependence of road network based on watershed scale-Take Shiyang River Basin as an example. Human Geography, 2015, 30(04): 123-128.

[3] Xu XC. Accurate understanding of China's current GDP accounting. Statistical Research, 2019, 36(05): 3-15.

[4] Wang SJ, Zhao J. Analysis of regional economic time-space disparities of Gansu province based on GIS. Science of Surveying and Mapping, 2010, 35(2): 170-172.

[5] Lv YQ, Lv P. Using Cobb Douglas production function model analysis of the effectiveness of R\&D activities. Statistical Research, 2013, 30(04) : 52-56 